\title{
A NEWTON-TYPE METHOD AND ITS APPLICATION
}

\author{
V. ANTONY VIJESH AND P. V. SUBRAHMANYAM \\ Received 6 March 2006; Accepted 26 March 2006
}

We prove an existence and uniqueness theorem for solving the operator equation $F(x)+$ $G(x)=0$, where $F$ is a continuous and Gâteaux differentiable operator and the operator $G$ satisfies Lipschitz condition on an open convex subset of a Banach space. As corollaries, a recent theorem of Argyros (2003) and the classical convergence theorem for modified Newton iterates are deduced. We further obtain an existence theorem for a class of nonlinear functional integral equations involving the Urysohn operator.

Copyright (c) 2006 Hindawi Publishing Corporation. All rights reserved.

\section{Introduction}

This paper considers the problem of approximating a locally unique solution $x^{*}$ of the equation $F(x)+G(x)=0$, where $F$ and $G$ are continuous operators defined on an open convex subset $D$ of a Banach space $X$ with values in a Banach space $Y$. The solution is obtained as the limit of a sequence of Newton-type iterates given by

$$
x_{n+1}=x_{n}-\left(F_{x_{n}}^{\prime}\right)^{-1}\left[F\left(x_{n}\right)+G\left(x_{n}\right)\right] \text {. }
$$

It may be noted that for $G \equiv 0,(1.1)$ reduces to Newton's method of iterates whose convergence is proved under the usual hypotheses that $F$ is Fréchet differentiable and $F_{x}^{\prime}$ is invertible. Recently, Argyros [3] obtained an interesting generalization of Newton's method which is further extended in this paper along with an earlier result of Vijesh and Subrahmanyam [1]. More specifically, we prove the convergence of a sequence of Newton-type iterates under mild conditions on $F$. In particular, the Gâteaux differentiable operator $F$ is assumed to satisfy the inequality $\left\|\left(F_{x_{0}}^{\prime}\right)^{-1}\left(F_{x}^{\prime}-F_{x_{0}}^{\prime}\right)\right\| \leq \epsilon$ in a certain neighbourhood $N\left(x_{0}\right)$ of $x_{0}$ while $G$ is required to be a contraction in $N\left(x_{0}\right)$. Using the main theorem, the existence of a unique continuous solution to a nonlinear functional integral equation involving the Urysohn operator is deduced from a corollary to the main theorem. This seems to be the first application of Newton-type algorithm to such nonlinear equations. Illustrative examples are also discussed. 
A Newton-type method and its application

\section{Convergence analysis}

Theorem 2.1 is a general theorem on the convergence of Newton-type iterates, proved under mild assumptions. It generalizes the theorems in $[1,3]$.

Theorem 2.1. Let $F$ and $G$ be continuous operators defined on an open convex subset $D$ of a Banach space $X$ with values in a Banach space $Y$. Suppose that $F$ is Gâteaux differentiable at each point in some neighbourhood of $x_{0} \in D$ and $G$ is Lipschitz on $D$. Assume further that

(i) $\left(F_{x_{0}}^{\prime}\right)^{-1} \in L(Y, X)$, the space of bounded linear operators from $Y$ to $X$;

(ii) for some $\eta \geq 0,\left\|\left(F_{x o}^{\prime}\right)^{-1}\left[F\left(x_{0}\right)+G\left(x_{0}\right)\right]\right\| \leq \eta$;

(iii) for some $r>0,\left\|\left(F_{x_{0}}^{\prime}\right)^{-1}\left[F_{x}^{\prime}-F_{x_{0}}^{\prime}\right]\right\| \leq \epsilon(\epsilon>0)$ whenever $x \in U\left(x_{0}, r\right)$, where $U\left(x_{0}\right.$, $r)=\left\{x \in B:\left\|x-x_{0}\right\|<r\right\}$ and $\|G(x)-G(y)\| \leq k\|x-y\|$ for $x, y \in D$ such that $3 \epsilon+\epsilon^{*}<1$ and $\left(1+c_{0} /(1-c)\right) \eta<r$, where $\epsilon^{*}=k\left\|\left(F_{x_{0}}^{\prime}\right)^{-1}\right\|, c_{0}=\left(\epsilon+\epsilon^{*}\right) /(1-\epsilon)$, and $c=\left(2 \epsilon+\epsilon^{*}\right) /(1-\epsilon)$;

(iv) $F_{x}^{\prime}$ is piecewise hemicontinuous for each $x \in U\left(x_{0}, r\right)$ and $\bar{U}\left(x_{0}, r\right) \subset D$.

Then the sequence $x_{n}(n \geq 0)$ generated recursively by (1.1) is well defined, remains in $U\left(x_{0}, r\right)$ for all $n \geq 0$, and converges to a unique solution $x^{*} \in \bar{U}\left(x_{0}, r\right)$ of the equation $F(x)+G(x)=0$. Moreover, the following error bounds hold for all $n \geq 2$ :

$$
\begin{aligned}
\left\|x_{n+1}-x_{n}\right\| & \leq c^{n-1} c_{0} \eta, \\
\left\|x_{n}-x^{*}\right\| & \leq \frac{c^{n-1}}{1-c} c_{0} \eta .
\end{aligned}
$$

Proof. Clearly by (ii) $\left\|x_{1}-x_{0}\right\|=\left\|\left(F_{x_{0}}^{\prime}\right)^{-1}\left[F\left(x_{0}\right)+G\left(x_{0}\right)\right]\right\| \leq \eta<r$, and hence $x_{1} \in$ $U\left(x_{0}, r\right)$. It follows from the choice of $\epsilon$ and the well-known Banach lemma (see Rall [4, page 50]) that $\left(F_{x_{1}}^{\prime}\right)^{-1}$ exists and $\left\|\left(F_{x_{1}}^{\prime}\right)^{-1} F_{x_{0}}^{\prime}\right\| \leq 1 /(1-\epsilon)$. Moreover (1.1) gives for $n=1$ that

$$
\begin{aligned}
x_{2}-x_{1}= & -\left(F_{x_{1}}^{\prime}\right)^{-1}\left[F\left(x_{1}\right)+G\left(x_{1}\right)\right] \\
= & -\left(F_{x_{1}}^{\prime}\right)^{-1} F_{x_{0}}^{\prime}\left(F_{x_{0}}^{\prime}\right)^{-1}\left[F\left(x_{1}\right)+G\left(x_{1}\right)-F\left(x_{0}\right)-G\left(x_{0}\right)+F\left(x_{0}\right)+G\left(x_{0}\right)\right] \\
= & -\left(F_{x_{1}}^{\prime}\right)^{-1} F_{x_{0}}^{\prime}\left(F_{x_{0}}^{\prime}\right)^{-1} \\
& \times\left\{\int_{0}^{1}\left[F_{\theta x_{1}+(1-\theta) x_{0}}^{\prime}-F_{x_{0}}^{\prime}\right]\left(x_{1}-x_{0}\right) d \theta+G\left(x_{1}\right)-G\left(x_{0}\right)\right\} \quad \text { (using (iv)) } \\
= & -\left(F_{x_{1}}^{\prime}\right)^{-1} F_{x_{0}}^{\prime} \\
& \times\left\{\int_{0}^{1}\left(F_{x_{0}}^{\prime}\right)^{-1}\left[F_{\theta x_{1}+(1-\theta) x_{0}}^{\prime}-F_{x_{0}}^{\prime}\right]\left(x_{1}-x_{0}\right) d \theta+\left(F_{x_{0}}^{\prime}\right)^{-1}\left[G\left(x_{1}\right)-G\left(x_{0}\right)\right]\right\} .
\end{aligned}
$$

So

$$
\begin{aligned}
\left\|x_{2}-x_{1}\right\| \leq & \left\|\left(F_{x_{1}}^{\prime}\right)^{-1} F_{x_{0}}^{\prime}\right\| \\
& \times\left\{\int_{0}^{1}\left\|\left(F_{x_{0}}^{\prime}\right)^{-1}\left[F_{\theta x_{1}+(1-\theta) x_{0}}^{\prime}-F_{x_{0}}^{\prime}\right]\left(x_{1}-x_{0}\right) d \theta\right\|+\left\|\left(F_{x_{0}}^{\prime}\right)^{-1}\left[G\left(x_{1}\right)-G\left(x_{0}\right)\right]\right\|\right\} \\
\leq & \frac{1}{1-\epsilon}\left\{\epsilon\left\|x_{1}-x_{0}\right\|+\left\|\left(F_{x_{0}}^{\prime}\right)^{-1}\right\| k\left\|x_{1}-x_{0}\right\|\right\}
\end{aligned}
$$




$$
\begin{aligned}
& =\frac{1}{1-\epsilon}\left\{\epsilon\left\|x_{1}-x_{0}\right\|+\epsilon^{*}\left\|x_{1}-x_{0}\right\|\right\}=\frac{\epsilon+\epsilon^{*}}{1-\epsilon}\left\|x_{1}-x_{0}\right\|, \\
\left\|x_{2}-x_{0}\right\| & \leq\left\|x_{2}-x_{1}\right\|+\left\|x_{1}-x_{0}\right\| \leq c_{0} \eta+\eta<r .
\end{aligned}
$$

Thus $x_{2} \in U\left(x_{0}, r\right)$. Again by Banach's lemma (see [4]) $\left(F_{x_{2}}^{\prime}\right)^{-1}$ exists and $\left\|\left(F_{x_{2}}^{\prime}\right)^{-1} F_{x_{0}}^{\prime}\right\| \leq$ $1 /(1-\epsilon)$. Assume that

$$
x_{k} \in U\left(x_{0}, r\right), \quad\left\|x_{k+1}-x_{k}\right\| \leq c^{k-1} c_{0} \eta \quad \text { for } k=2,3, \ldots, n-1 .
$$

In view of hypotheses (iii) and (iv), it follows as before from Banach's lemma that $\left(F_{x_{n}}^{\prime}\right)^{-1}$ exists and

$$
\left\|\left(F_{x_{n}}^{\prime}\right)^{-1} F_{x_{0}}^{\prime}\right\| \leq \frac{1}{1-\epsilon}
$$

By hypotheses (iii) and (iv) and (2.5), we obtain

$$
\begin{aligned}
& x_{n+1}-x_{n} \\
& =-\left(F_{x_{n}}^{\prime}\right)^{-1}\left(F\left(x_{n}\right)+G\left(x_{n}\right)\right) \\
& =-\left(F_{x_{n}}^{\prime}\right)^{-1} F_{x_{0}}^{\prime}\left(F_{x_{0}}^{\prime}\right)^{-1}\left(F\left(x_{n}\right)+G\left(x_{n}\right)\right) \\
& =-\left(F_{x_{n}}^{\prime}\right)^{-1} F_{x_{0}}^{\prime}\left(F_{x_{0}}^{\prime}\right)^{-1} \\
& \times\left[F\left(x_{n}\right)+G\left(x_{n}\right)-F\left(x_{n-1}\right)-G\left(x_{n-1}\right)+F\left(x_{n-1}\right)+G\left(x_{n-1}\right)\right] \\
& =-\left(F_{x_{n}}^{\prime}\right)^{-1} F_{x_{0}}^{\prime}\left(F_{x_{0}}^{\prime}\right)^{-1} \\
& \times\left\{\int_{0}^{1}\left(\left[F_{\theta x_{n}+(1-\theta) x_{n-1}}^{\prime}-F_{x_{0}}^{\prime}\right]+\left[F_{x_{0}}^{\prime}-F_{x_{n-1}}^{\prime}\right]\right)\left(x_{n}-x_{n-1}\right) d \theta+G\left(x_{n}\right)-G\left(x_{n-1}\right)\right\} \\
& =-\left(F_{x_{n}}^{\prime}\right)^{-1} F_{x_{0}}^{\prime}\left\{\int_{0}^{1}\left(F_{x_{0}}^{\prime}\right)^{-1}\left(\left[F_{\theta x_{n}+(1-\theta) x_{n-1}}^{\prime}-F_{x_{0}}^{\prime}\right]+\left[F_{x_{0}}^{\prime}-F_{x_{n-1}}^{\prime}\right]\right)\left(x_{n}-x_{n-1}\right) d \theta\right. \\
& \left.+\left(F_{x_{0}}^{\prime}\right)^{-1}\left[G\left(x_{n}\right)-G\left(x_{n-1}\right)\right]\right\}, \\
& \left\|x_{n+1}-x_{n}\right\| \leq\left\|\left(F_{x_{n}}^{\prime}\right)^{-1} F_{x_{0}}^{\prime}\right\|\left\{\int_{0}^{1}\left\|\left(F_{x_{0}}^{\prime}\right)^{-1}\left[F_{\theta x_{n}+(1-\theta) x_{n-1}}^{\prime}-F_{x_{0}}^{\prime}\right]\right\|\left\|\left(x_{n}-x_{n-1}\right)\right\| d \theta\right. \\
& +\int_{0}^{1}\left\|\left(F_{x_{0}}^{\prime}\right)^{-1}\left[F_{x_{0}}^{\prime}-F_{x_{n-1}}^{\prime}\right]\right\|\left\|\left(x_{n}-x_{n-1}\right)\right\| d \theta \\
& \left.+\left\|\left(F_{x_{0}}^{\prime}\right)^{-1}\right\| k\left\|x_{n}-x_{n-1}\right\|\right\} \\
& \left\|x_{n+1}-x_{n}\right\| \leq \frac{2 \epsilon+\epsilon^{*}}{1-\epsilon}\left\|x_{n}-x_{n-1}\right\|
\end{aligned}
$$


4 A Newton-type method and its application

Thus by induction hypothesis (2.4), $\left\|x_{n+1}-x_{n}\right\| \leq c^{n-1} c_{0} \eta$. Since

$$
\begin{aligned}
\left\|x_{n+1}-x_{0}\right\| & \leq\left\|x_{n+1}-x_{n}\right\|+\left\|x_{n}-x_{n-1}\right\|+\cdots+\left\|x_{1}-x_{0}\right\| \\
& \leq c^{n-1} c_{0} \eta+c^{n-2} c_{0} \eta+\cdots+c c_{0} \eta+c_{0} \eta+\eta, \\
\left\|x_{n+1}-x_{0}\right\| & \leq \eta\left[1+c_{0} \frac{1-c^{n}}{1-c}\right] \leq \eta\left[1+\frac{c_{0}}{1-c}\right]<r .
\end{aligned}
$$

Hence $x_{n} \in U\left(x_{0}, r\right)$ for all $n \geq 0$. For $k \geq 2, m \geq 0$.

$$
\begin{aligned}
\left\|x_{k+m}-x_{k}\right\| & \leq\left\|x_{k+m}-x_{k+m-1}\right\|+\left\|x_{k+m-1}-x_{k+m-2}\right\|+\cdots+\left\|x_{k+1}-x_{k}\right\| \\
& \leq c^{k+m-2} c_{0} \eta+c^{k+m-3} c_{0} \eta+\ldots+c^{k-1} c_{0} \eta \\
& \leq \eta c_{0} c^{k-1}\left[1+c+\cdots+c^{m-1}\right] \\
& \leq \frac{1-c^{m}}{1-c} c^{k-1} c_{0} \eta \leq \frac{c^{k-1}}{1-c} c_{0} \eta .
\end{aligned}
$$

As $0<c<1, x_{n}$ is a Cauchy sequence in the closed subset $\bar{U}\left(x_{0}, r\right)$ of the Banach space $X$; it hence converges to an element $x^{*}$ in $\bar{U}\left(x_{0}, r\right)$. From hypothesis (iii) using triangle inequality, it follows that $\left\|F_{x_{n}}^{\prime}\right\| \leq M$, where $M=\left(\epsilon /\left\|\left(F_{x_{0}}^{\prime}\right)^{-1}\right\|+\left\|\left(F_{x_{0}}^{\prime}\right)^{-1}\right\|\right)$. Since

$$
x_{n+1}=x_{n}-\left(F_{x_{n}}^{\prime}\right)^{-1}\left(F\left(x_{n}\right)+G\left(x_{n}\right)\right), \quad F\left(x_{n}\right)+G\left(x_{n}\right)=-F_{x_{n}}^{\prime}\left(x_{n+1}-x_{n}\right) .
$$

So

$$
\left\|F\left(x_{n}\right)+G\left(x_{n}\right)\right\| \leq\left\|F_{x_{n}}^{\prime}\right\|\left\|x_{n+1}-x_{n}\right\| \leq M\left\|x_{n+1}-x_{n}\right\| .
$$

Proceeding to the limit in (2.10) as $n$ tends to infinity and using the continuity of $F$ and $G$ it follows from the convergence of $\left(x_{n}\right)$ to $x^{*}$ that $F\left(x^{*}\right)+G\left(x^{*}\right)=0$. If $x^{*}$ and $y^{*}$ are two solutions of $F(x)+G(x)=0$ in $\bar{U}\left(x_{0}, r\right)$, then

$$
\begin{aligned}
\left\|x^{*}-y^{*}\right\|= & \left\|x^{*}-y^{*}-\left(F_{x_{0}}^{\prime}\right)^{-1}\left[F\left(x^{*}\right)+G\left(x^{*}\right)-F\left(y^{*}\right)-G\left(y^{*}\right)\right]\right\| \\
\leq & \left\|\int_{0}^{1}\left[I-\left(F_{x_{0}}^{\prime}\right)^{-1} F_{\theta x^{*}+(1-\theta) y^{*}}^{\prime}\right]\left(x^{*}-y^{*}\right) d \theta\right\| \\
& +\left\|\left(F_{x_{0}}^{\prime}\right)^{-1}\left(G\left(x^{*}\right)-G\left(y^{*}\right)\right)\right\| \\
\leq & \epsilon\left\|x^{*}-y^{*}\right\|+\epsilon^{*}\left\|x^{*}-y^{*}\right\| \\
= & \left(\epsilon+\epsilon^{*}\right)\left\|x^{*}-y^{*}\right\|<\left\|x^{*}-y^{*}\right\| \quad \text { as } 0 \leq \epsilon+\epsilon^{*} \leq 3 \epsilon+\epsilon^{*}<1 .
\end{aligned}
$$

This contradiction implies that $x^{*}=y^{*}$. Hence the theorem holds.

Corollary 2.2 (see Argyros [3, Theorem 1]). Let F be a continuous operator defined on an open convex subset $D$ of a Banach space $X$ with values in a Banach space $Y$ and continuously Fréchet differentiable at some $x_{0} \in D$. Assume that

(i) $\left(F_{x_{0}}^{\prime}\right)^{-1} \in L(Y, X)$;

(ii) there exists a parameter $\eta$ such that $0 \leq\left\|\left(F_{x_{0}}^{\prime}\right)^{-1} F\left(x_{0}\right)\right\| \leq \eta$; 
(iii) for some $\epsilon \in(0,1 / 3)$, there exists $\delta>0$ such that $\left\|\left(F_{x_{0}}^{\prime}\right)^{-1}\left(F_{x}^{\prime}-F_{x_{0}}^{\prime}\right)\right\| \leq \epsilon$ whenever $x \in U\left(x_{0}, \delta\right)=\left\{x \in X:\left\|x-x_{0}\right\|<\delta\right\}$, with $\left(c^{2} /(1-c)+c_{0}+1\right) \eta<\delta$, where $c_{0}=$ $\epsilon /(1-\epsilon)$ and $c=2 c_{0}$.

Then the Newton iterates $\left(x_{n}\right)$ generated by (1.1) are well defined, remain in $U\left(x_{0}, \delta\right)$ for all $n \geq 0$, and converge to a solution $x^{*} \in \bar{U}\left(x_{0}, \delta\right)$ of equation $F(x)=0$. Moreover, for all $n \geq 2$, the following error bounds hold:

$$
\begin{gathered}
\left\|x_{n+1}-x_{n}\right\| \leq c^{n}\left\|x_{1}-x_{0}\right\|, \\
\left\|x_{n}-x^{*}\right\| \leq \frac{c^{n}}{1-c}\left\|x_{1}-x_{0}\right\| .
\end{gathered}
$$

Proof. Since $F$ is Fréchet differentiable, it is Gâteaux differentiable. Take $G \equiv 0$ in Theorem 2.1. Let $\delta=r$ and note that

$$
\delta>\left[\frac{c^{2}}{1-c}+c_{0}+1\right] \eta \geq\left[1+\frac{c_{0}}{1-c}\right] \eta .
$$

Since $F$ is continuously Fréchet differentiable, $F_{x}^{\prime}$ is hemicontinuous at each $x \in U\left(x_{0}, \delta\right)$ and thus all the conditions of Theorem 2.1 are satisfied. So $F$ has a unique zero in $\bar{U}\left(x_{0}, \delta\right)$.

Corollary 2.3. Let $F$ be a continuous operator defined on an open convex subset $D$ of a Banach space $X$ with values in a Banach space $Y$ having a Gâteaux derivative at each point in some neighbourhood of $x_{0} \in D$. Assume further that

(i) the inverse of $F_{x_{o}}^{\prime}$ exists and $\left(F_{x_{0}}^{\prime}\right)^{-1} \in L(Y, X)$;

(ii) for some $\eta \geq 0\left\|\left(F_{x_{0}}^{\prime}\right)^{-1} F\left(x_{0}\right)\right\| \leq \eta$;

(iii) for some $r>0,\left\|\left(F_{x_{0}}^{\prime}\right)^{-1}\left[F_{x}^{\prime}-F_{x_{0}}^{\prime}\right]\right\| \leq \epsilon$ whenever $x \in U\left(x_{0}, r\right)$. Suppose $\left(1+c_{0} /\right.$ $(1-c)) \eta<r$ and $0<3 \epsilon<1$, where $c_{0}=\epsilon /(1-\epsilon)$ and $c=2 c_{0}$;

(iv) $F_{x}^{\prime}$ is piecewise hemi continuous at each $x \in U\left(x_{0}, r\right)$.

Then the modified Newton iterates $\left(x_{n}\right), n \geq 0$, generated by

$$
x_{n+1}=x_{n}-\left(F_{x_{o}}^{\prime}\right)^{-1} F\left(x_{n}\right)
$$

are well defined, remain in $U\left(x_{0}, r\right)$ for all $n \geq 0$, and converge to a solution $x^{*} \in \bar{U}\left(x_{0}, r\right)$ of $F(x)=0$.

To prove Corollary 2.3, set $G \equiv 0$ in Theorem 2.1 and proceed as in Theorem 2.1.

\section{Solutions of a class of nonlinear functional integral equations}

In this section, some existence theorems for an operator equation involving the Urysohn operator are proved. More specifically, given a compact subset $\Omega$ of $\mathbb{R}, g \in C(\Omega \times \mathbb{R})$ and $K \in C\left(\Omega^{2} \times \mathbb{R}\right)$, Theorem 3.2 gives sufficient conditions for the existence of a unique solution of the nonlinear functional integral equation of the form

$$
x(t)+\int_{\Omega} K(t, s, x(s)) d s+g(t, x(t))=0 \quad \forall t \in \Omega, x \in C(\Omega) .
$$

Hereafter we denote $x(t)+\int_{\Omega} K(t, s, x(s)) d s$ by $F x(t)$. 
Lemma 3.1. For $K \in C\left(\Omega^{2} \times \mathbb{R}\right)$, suppose that $K_{3}(t, s, u)=\partial K / \partial u \in C\left(\Omega^{2} \times \mathbb{R}\right)$ satisfies the Lipschitz condition with respect to the third variable. Then the Fréchet derivative of $F$ exists for $x \in U\left(x_{0}, r\right)$ and $x_{0} \in C(\Omega)$ and the derivative at $x$ is given by

$$
F_{x}^{\prime}(h) t=h(t)+\int_{\Omega} K_{3}(t, s, x(s)) h(s) d s .
$$

For the proof, see [2].

Theorem 3.2. Let $K(t, s, u) \in C\left(\Omega^{2} \times \mathbb{R}\right)$, let $K_{3}(t, s, u) \in C\left(\Omega^{2} \times \mathbb{R}\right)$, let $g \in C(\Omega \times \mathbb{R})$, and let $\Omega$ be a compact subset of $\mathbb{R}$ whose Lebesgue measure is equal to $d>0$. Suppose that

(i) for some $m \in(0,1),\left\|K_{3}\left(t, s, u_{1}\right)-K_{3}\left(t, s, u_{2}\right)\right\| \leq m\left\|u_{1}-u_{2}\right\|$ holds for all $\left(t, s, u_{i}\right) \in$ $\Omega^{2} \times \mathbb{R}, i=1,2$;

(ii) $\left|g\left(t, y_{1}\right)-g\left(t, y_{2}\right)\right| \leq \epsilon^{*}\left|y_{1}-y_{2}\right|$ for all $y_{1}, y_{2} \in \mathbb{R}, t \in \Omega$, where $\beta+\epsilon^{*}<1 \beta=$ $\sup _{t \in \Omega}\left|\int_{\Omega} K_{3}\left(t, s, x_{0}(s)\right) d s\right|$. Further let $\eta \geq 0$ be chosen such that $\eta \geq \sup _{t \in \Omega} \mid$ $F x_{0}(t)+g\left(t, x_{0}(t)\right) \mid$

(iii) let $\alpha_{1}$ and $\alpha_{2}$ be the positive roots of $p(x)=4 m^{2} d^{2} x^{2}-4 m d(1-\beta)\left(2-2 \beta-\epsilon^{*}\right) x+$ $\left[\left(1-\beta-\epsilon^{*}\right)(1-\beta)\right]^{2}$ and let $r_{1}$ and $r_{2}$ with $r_{1} \leq r_{2}$ be the positive roots of $q(x)=$ $3 m d(1-\beta) x^{2}-\left[\left(1-\beta-\epsilon^{*}\right)(1-\beta)+2 m d \eta\right] x+(1-\beta) \eta$;

(iv) suppose further that $\eta \leq \min \left\{\alpha_{1}, \alpha_{2}\right\}$ and $\eta /(1-\beta)<r_{1}<\left(1-\beta-\epsilon^{*}\right) / 3 m d$.

Then the functional integral equation $F x(t)+g(t, x(t))=0$ for all $t \in \Omega$ has a unique solution in $\bar{U}\left(x_{0}, r_{0}\right)$ for all $r_{0} \in\left(r_{1}, \min \left\{r_{2},\left(1-\beta-\epsilon^{*}\right) / 3 m d\right\}\right)$ and the sequence of iterates given in (1.1) converges to this solution.

Proof. Clearly $F$ maps $C(\Omega)$ into itself. Let $G: C(\Omega) \rightarrow C(\Omega)$ be defined by $G x(t)=$ $g(t, x(t)) \forall t \in \Omega$. Then $G$ maps $C(\Omega)$ into itself,

$$
\begin{aligned}
\left|G x_{1}(t)-G x_{2}(t)\right| & =\left|g\left(t, x_{1}(t)\right)-g\left(t, x_{2}(t)\right)\right| \\
& \leq \epsilon^{*}\left|x_{1}(t)-x_{2}(t)\right| \quad(\text { by (ii) }) \\
& \leq \epsilon^{*}|| x_{1}-x_{2}|| .
\end{aligned}
$$

Thus $\left\|G x_{1}-G x_{2}\right\| \leq \epsilon^{*}\left\|x_{1}-x_{2}\right\|$. Assumption (i) together with Lemma 3.1 implies that $F_{x}^{\prime}$ exists. Now,

$$
\begin{aligned}
\left|\left(I-F_{x_{0}}^{\prime}\right) h(t)\right| & =\left|h(t)-h(t)-\int_{\Omega} K_{3}\left(t, s, x_{0}(s)\right) h(s) d s\right| \\
& \leq \int_{\Omega}\left|K_{3}\left(t, s, x_{0}(s)\right) h(s) d s\right| \leq\|h\| \int_{\Omega}\left\|K_{3}\left(t, s, x_{0}(s)\right)\right\| d s \\
& \leq\|h\| \beta .
\end{aligned}
$$

This implies that $\left\|I-F_{x_{0}}^{\prime}\right\| \leq \beta<1$, and hence $F_{x_{0}}^{\prime}$ is invertible and by Banach's lemma, $\left\|\left(F_{x_{0}}^{\prime}\right)^{-1}\right\| \leq 1 /(1-\beta)$. Since the roots $\alpha_{1}$ and $\alpha_{2}$ of $p(x)$ are positive and

$$
\left(x-\alpha_{1}\right)\left(x-\alpha_{2}\right)=x^{2}-\left[\frac{(1-\beta)\left(2-2 \beta-\epsilon^{*}\right)}{m d}\right] x+\left[\frac{\left(1-\beta-\epsilon^{*}\right)(1-\beta)}{2 m d}\right]^{2},
$$


by assumption (iv), $\left(\eta-\alpha_{1}\right)\left(\eta-\alpha_{2}\right) \geq 0$. This implies that

$$
\eta^{2}-\left[\frac{(1-\beta)\left(2-2 \beta-\epsilon^{*}\right)}{m d}\right] \eta+\left[\frac{\left(1-\beta-\epsilon^{*}\right)(1-\beta)}{2 m d}\right]^{2} \geq 0
$$

Thus

$$
\left[\left(1-\beta-\epsilon^{*}\right)(1-\beta)\right]^{2}-4 m d(1-\beta)\left(2-2 \beta-\epsilon^{*}\right) \eta+4 m^{2} d^{2} \eta^{2} \geq 0
$$

In other words, the discriminant of the quadratic equation $q(x)=0$ being

$$
\left[\left(1-\beta-\epsilon^{*}\right)(1-\beta)+2 m d \eta\right]^{2}-12 m d(1-\beta)^{2} \eta
$$

is nonnegative by (3.6). So $q(x)$ always has positive roots $r_{1}$ and $r_{2}$. Since by assumption (iv) $\eta /(1-\beta)<r_{1}<\left(1-\beta-\epsilon^{*}\right) / 3 m d$, choose $r_{0}$ with $r_{1}<r_{0}<\min \left\{r_{2},\left(1-\beta-\epsilon^{*}\right) /\right.$ $3 m d\}$. Then

$$
q\left(r_{0}\right)=3 m d(1-\beta) r_{0}^{2}-r_{0}\left[\left(1-\beta-\epsilon^{*}\right)(1-\beta)+2 m d \eta\right]+(1-\beta) \eta<0
$$

So

$$
\left[\left(1-\beta-\epsilon^{*}\right)(1-\beta)+2 m d \eta\right] r_{0}-3 m d(1-\beta) r_{0}^{2}>(1-\beta) \eta
$$

Hence

$$
\left[\frac{1-\beta-2 \mathrm{mr}_{0} d}{1-\beta-\epsilon^{*}-3 \mathrm{mr}_{0} d}\right] \frac{\eta}{1-\beta}<r_{0}
$$

Now for $t \in \Omega$,

$$
\begin{aligned}
\left|\left(F_{x_{0}}^{\prime}\right)^{-1}\left(F_{x}^{\prime}-F_{x_{0}}^{\prime}\right) h(t)\right| & =\left|\left(F_{x_{0}}^{\prime}\right)^{-1}\left\{\int_{\Omega}\left(K_{3}(t, s, x(s))-K_{3}\left(t, s, x_{0}(s)\right)\right) h(s) d s\right\}\right| \\
& \leq\left\|\left(F_{x_{0}}^{\prime}\right)^{-1}\right\| \int_{\Omega}\left|K_{3}(t, s, x(s))-K_{3}\left(t, s, x_{0}(s)\right)\right||h(s)| d s \\
& \leq \frac{\operatorname{mr}_{0} d}{1-\beta}\|h\| \quad(\text { using (i) }) .
\end{aligned}
$$

This implies that $\left\|\left(F_{x_{0}}^{\prime}\right)^{-1}\left(F_{x}^{\prime}-F_{x_{0}}^{\prime}\right)\right\| \leq \operatorname{mr}_{0} d /(1-\beta)$. Setting $\epsilon=\mathrm{mr}_{0} d /(1-\beta), \epsilon_{1}^{*}=$ $\epsilon^{*} /(1-\beta), \eta_{1}=\eta /(1-\beta), c_{0}=\left(\epsilon+\epsilon_{1}^{*}\right) /(1-\epsilon)$, and $c=\left(2 \epsilon+\epsilon_{1}^{*}\right) /(1-\epsilon)$, and using (3.11), all the hypotheses of Theorem 2.1 are readily verified. Hence $F x(t)+g(t, x(t))=$ 0 for all $t \in \Omega$ has a unique solution in $\bar{U}\left(x_{0}, r_{0}\right)$. Thus there is a unique continuous 
function $x^{*}$ satisfying

$$
x^{*}(t)+\int_{\Omega} K\left(t, s, x^{*}(s)\right) d s+g\left(t, x^{*}(t)\right)=0, \quad \forall t \in \Omega,
$$

and this solution can obtained as the limit of the iterates in (1.1).

\section{Illustrative examples}

The example below illustrates Theorem 3.2.

Example 4.1. Consider the problem of solving the functional integral equation

$$
x(t)-\frac{1}{1000} \int_{0}^{1} t \cos (s t) \sin \left(\frac{x(s)-1}{1000}\right) d s-\sin \left(\frac{|x(t)|}{1000}\right)=0 \quad \text { in } C[0,1] .
$$

For the choice $F(x)=x(t)-1 / 1000 \int_{0}^{1} t \cos (\operatorname{st}) \sin ((x(s)-1) / 1000) d s$ and $g(t, x(t))=$ $-\sin (|x(t)| / 1000)$, where $\Omega=[0,1]$ and $x_{0} \equiv 1 / 1000, \beta=\sup _{t \in \Omega}\left|F x_{0}(t)+g\left(t, x_{0}(t)\right)\right|$ $=\sup _{t \in \Omega}\left|1 / 1000-0.17 \times 10^{-7} \sin t-0.17 \times 10^{-7}\right|<1 / 1000, F_{x}^{\prime} h(t)=h(t)-1 / 1000^{2} \int_{0}^{1} t$ $\cos (\mathrm{st}) \cos ((x(s)-1) / 1000) h(s) d s$. For $\eta=1 / 1000, \eta /(1-\beta)=0.001000001$. Setting $m=10^{-6}, \epsilon^{*}=10^{-3}$, and $d=1$, we have $p(x)=4 \times 10^{-12} x^{2}-0.000007993 x+$ 0.997997006 and $\alpha_{1}$ the smaller positive root of $p(x)$ is $0.000007993-0.000006923 /$ $8 \times 10^{-} 12=133750>\eta$. For $q(x)=0.000002999 x^{2}-0.998999002 x+0.00099999, r_{1}$ the smaller positive root of $q(x)$ is $(0.998999002-0.998998996) / 0.000005998=$ $0.001000333>\eta /(1-\beta)$. As the other positive root $r_{2}=338110.7032>332999.667=$ $\left(1-\beta-\epsilon^{*}\right) / 3 m d$, Theorem 3.2(4) is satisfied. For $r_{0} \in(0.001000333,332999.667)$, all the conditions of Theorem 3.2 are fulfilled. So the functional integral equation has a unique solution in $\bar{U}\left(x_{0}, r_{0}\right)$ and this solution can be obtained as the limit of the sequence of iterates in (1.1).

Example 4.2. The next example shows that Theorem 2.1 is more general than Corollary 2.2, the main theorem obtained by Argyros [3].

Let $f: \mathbb{R} \rightarrow \mathbb{R}$ be the map defined by

$$
f(x)= \begin{cases}\left(\frac{x-0.01}{25}\right)\left(20+x^{2} \sin \frac{1}{x}\right), & x \neq 0, \\ -\frac{1}{125}, & x=0,\end{cases}
$$

let $g: \mathbb{R} \rightarrow \mathbb{R}$ be the map defined by $g(x)=|x|-0.01 / 1000$. Choose $x_{0}=0, \epsilon=0.25$, and $r=0.4$. Clearly for $y \in \mathbb{R}$,

$$
f_{x}^{\prime}(y)= \begin{cases}{\left[\left(\frac{x-0.01}{25}\right)\left(2 x \sin \frac{1}{x}-\cos \frac{1}{x}\right)+\left(\frac{20+x^{2} \sin (1 / x)}{25}\right)\right] y,} & \text { for } x \neq 0 \\ 0.8 y, & \text { for } x=0\end{cases}
$$


$\left(f_{x_{0}}^{\prime}\right)^{-1}$ exists and $\left\|\left(f_{x_{0}}^{\prime}\right)^{-1}\right\|=1 / 0.8$. As $G$ satisfies the Lipschitz condition with $M=$ $1 / 1000, \epsilon^{*}=\left(M\left\|\left(f_{x_{0}}^{\prime}\right)^{-1}\right\|\right)=0.00125$. For $x \in(-0.4,0.4)$,

$$
\begin{aligned}
\left\|f_{x}^{\prime}-f_{x_{o}}^{\prime}\right\| & =\sup \left\{\left|\left(f_{x}^{\prime}-f_{x_{o}}^{\prime}\right)(y)\right|:\|y\| \leq 1\right\} \\
& =\sup \left\{\left|\left[\left(\frac{x-0.01}{25}\right)\left(2 x \sin \frac{1}{x}-\cos \frac{1}{x}\right)+\left(\frac{x^{2} \sin (1 / x)}{25}\right)\right] y\right|:\|y\| \leq 1\right\} \\
& \leq \sup \left\{\left[\frac{0.4+0.01}{25}(2 \times 0.4+1)+\frac{0.16}{25}\right]|y|:\|y\| \leq 1\right\} \\
& =0.03592 . \leq 0.25 \times 0.8=\frac{\epsilon}{\left\|\left(F_{x_{0}}^{\prime}\right)^{-1}\right\|},
\end{aligned}
$$

and $\left\|\left(F_{x_{0}}^{\prime}\right)^{-1}\left(F_{x}^{\prime}-F_{x_{0}}^{\prime}\right)\right\|<\epsilon=0.25$. Also $c_{0}=0.335, c=0.66833, \eta=0.0100125,0 \leq$ $\left\|\left(f_{x_{0}}^{\prime}\right)^{-1}\left(f\left(x_{0}\right)+g\left(x_{0}\right)\right)\right\| \leq 0.0100125$, and $\left(c_{0} /(1-c)+1\right)=2.0097891587$. Since $\left(c_{0} /(1-c)+1\right) \eta<r$, all the conditions of Theorem 2.1 are verified. Thus $f(x)+g(x)=0$ has a unique solution in $\bar{U}(0,0.4)$. However, $F_{x}^{\prime}$ is not continuous at zero but piecewise hemicontinuous in $\bar{U}(0,0.4)$. It may be noted that Corollary 2.2 (due to Argyros) cannot be applied to prove that this equation has a solution, whereas Theorem 2.1 insures this.

\section{Acknowledgment}

V. Antony Vijesh acknowledges the Council of Scientific and Industrial Research (India) for the financial support provided in the form of a Junior Research Fellowship to carry out this research work.

\section{References}

[1] V. Antony Vijesh and P. V. Subrahmanyam, An extension of Newton's method, Proceedings of the International Conference on Analysis and Applications, Allied Publishers Pvt., New Delhi, 2004, pp. $120-126$.

[2] J. Appell, E. De Pascale, and P. P. Zabrejko, On the application of the Newton-Kantorovich method to nonlinear integral equations of Uryson type, Numerical Functional Analysis and Optimization 12 (1991), no. 3-4, 271-283.

[3] I. K. Argyros, On a theorem of L. V. Kantorovich concerning Newton's method, Journal of Computational and Applied Mathematics 155 (2003), no. 2, 223-230.

[4] L. B. Rall, Computational Solution of Nonlinear Operator Equations, John Wiley \& Sons, New York, 1969.

V. Antony Vijesh: Department of Mathematics, Indian Institute of Technology Madras, Chennai 600036, India

E-mail address: antonykv@iitm.ac.in

P. V. Subrahmanyam: Department of Mathematics, Indian Institute of Technology Madras,

Chennai 600036, India

E-mail address: pvs@iitm.ac.in 


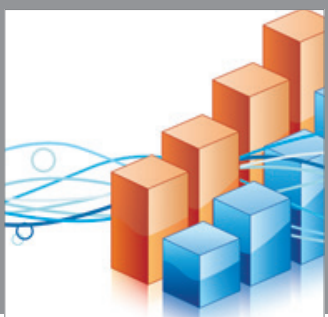

Advances in

Operations Research

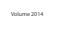

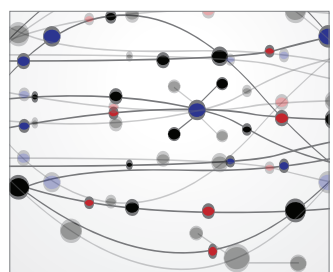

\section{The Scientific} World Journal
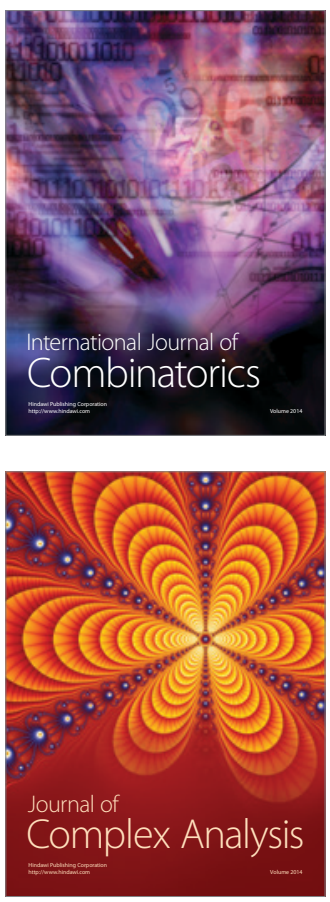

International Journal of

Mathematics and

Mathematical

Sciences
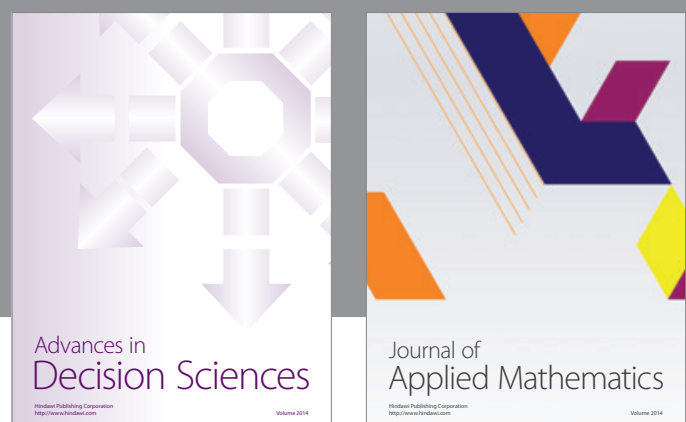

Journal of

Applied Mathematics
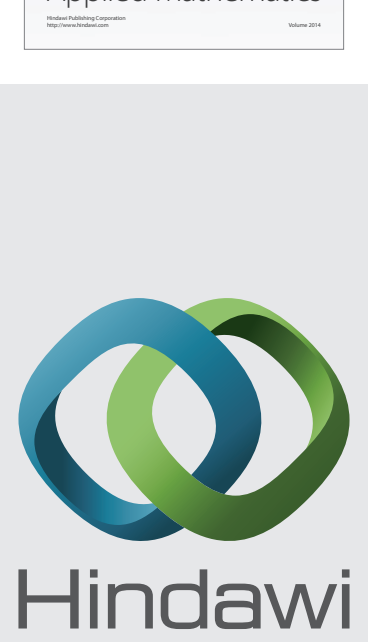

Submit your manuscripts at http://www.hindawi.com
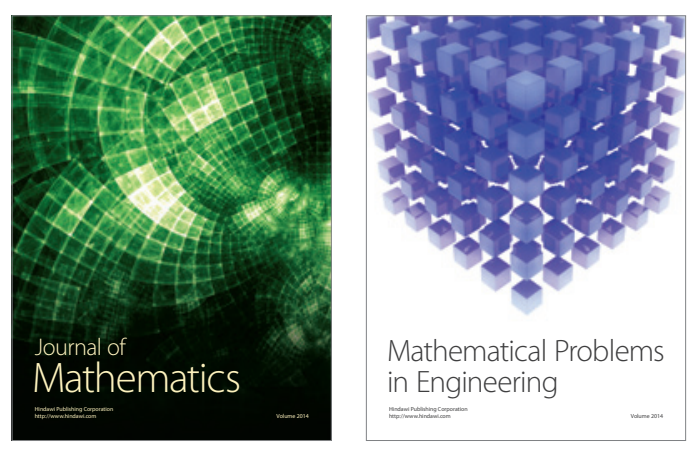

Mathematical Problems in Engineering
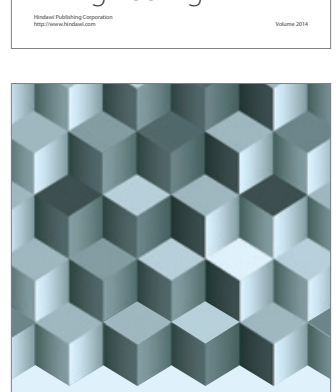

Journal of

Function Spaces
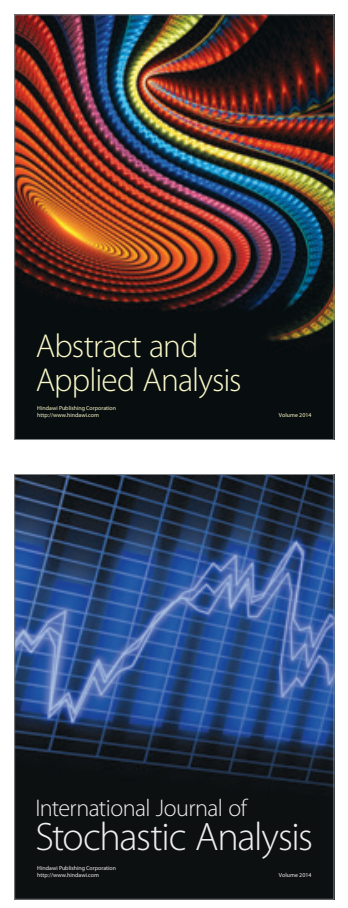

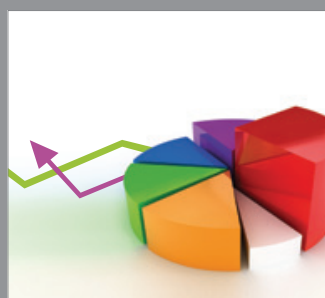

ournal of

Probability and Statistics

Promensencen
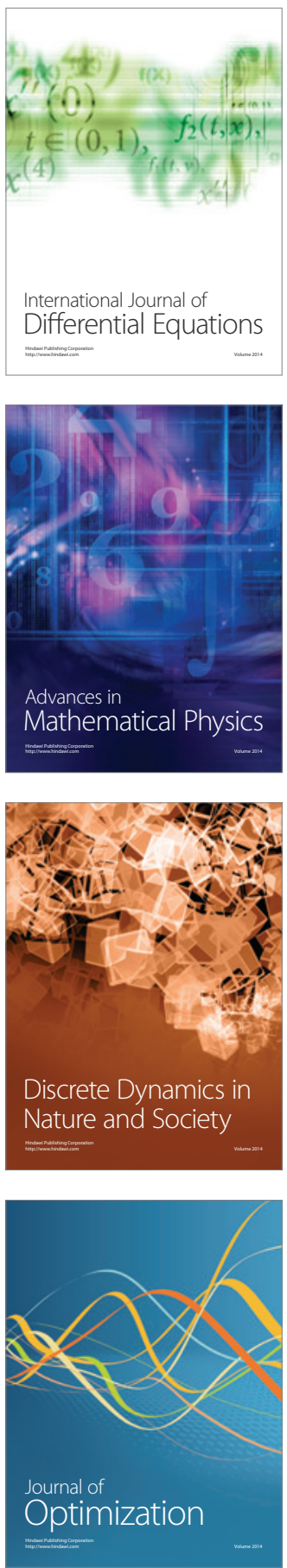